\title{
A Plenoptic Multi-Color Imaging Pyrometer
}

\author{
Paul M. Danehy, ${ }^{1}$ \\ NASA Langley Research Center, Hampton VA, 23681-2199 \\ William D. Hutchins, ${ }^{2}$ \\ Virginia Tech, Blacksburg VA, 24061 \\ Timothy Fahringer, ${ }^{3}$ and Brian S. Thurow ${ }^{4}$ \\ Auburn University, Auburn AL, 36849
}

\begin{abstract}
A three-color pyrometer has been developed based on plenoptic imaging technology. Three bandpass filters placed in front of a camera lens allow separate $2 \mathrm{D}$ images to be obtained on a single image sensor at three different and adjustable wavelengths selected by the user. Images were obtained of different black- or grey-bodies including a calibration furnace, a radiation heater, and a luminous sulfur match flame. The images obtained of the calibration furnace and radiation heater were processed to determine 2D temperature distributions. Calibration results in the furnace showed that the instrument can measure temperature with an accuracy and precision of 10 Kelvins between 1100 and $1350 \mathrm{~K}$. Timeresolved 2D temperature measurements of the radiation heater are shown.
\end{abstract}

\section{Introduction}

$\mathrm{O}$ ptical pyrometry - the use of an optical instrument to remotely measure temperature - has been used in countless applications where the light emission from a hot object is collected by a sensor and processed to measure temperature. Many commercially available pyrometers measure temperature at a single point. ${ }^{1}$ Such instruments can view the absolute intensity of the radiation at one or more wavelengths to determine the temperature. Some single-point instruments collect and disperse a wide range of the visible or infrared spectrum. Instruments that measure a wide spectral range collect more information about the radiation and can therefore be more reliable and accurate than simple one- or two-color pyrometers. Some instruments, typically near-infrared or mid-infrared cameras, measure two dimensional (2D) temperature distributions, usually by imaging a single color or wavelength band of radiation. While these instruments are very sensitive to relative changes in temperature, they can be difficult to calibrate and are susceptible to errors caused by changes in emissivity, surface finish, composition or phase change, transmission losses or absorption interferences since they rely on absolute intensity measurements. ${ }^{1}$ Hybrid instruments, for example, where a 2D camera is placed at the exit of an imaging spectrometer, can be used to measure fully resolved spectra at each point along a 1D line. The spectral resolution of such systems is advantageous because the full spectrum can be analyzed at each point and the temperature determined from the relative shape of the spectra. In such systems, interferences can be readily identified and avoided, but 2D spatial information is not obtained.

Some fully 2D, spectrally resolved systems exist as reviewed by Hagen and Kudenov. ${ }^{2}$ These systems are sometimes termed multi-spectral or hyperspectral imaging systems. Such systems have previously been used for optical pyrometry including three-color 2D imaging systems. ${ }^{3}$ Many of these optical systems which can provide 2D images at multiple wavelengths use beam splitters or other optical elements and are cumbersome, growing in size as more wavelengths are added. An alternate method is to mount filters on a mechanical disk (called a filter wheel) and these filters are sequentially rotated in front in a camera to acquire images at different wavelengths. Such filterwheel systems have several drawbacks: this bulky arrangement has moving parts which can fail, the images are

\footnotetext{
${ }^{1}$ Senior Researcher, Advanced Measurement and Data Systems Branch, MS 493, AIAA Associate Fellow.

${ }^{2}$ Undergraduate Student, Department of Physics.

${ }^{3}$ Graduate Research Assistant, AIAA Student member.

${ }^{4}$ W. Allen and Martha Reed Associate Professor, AIAA Associate Fellow.
} 
taken at different times, slowing data acquisition and preventing capture of transient events. This paper describes an alternative imaging technology known as a plenoptic imager, coupled with spectral filters ${ }^{4,5}$ to perform pyrometry.

A plenoptic camera is similar to a conventional greyscale camera except that a thin microlens array is placed in front of the sensor. ${ }^{6,7}$ The use of the microlens array allows measurement of additional information about the light collected by the primary lens including the angle of the incident rays as well as the intensity. This additional information allows ranging information to be obtained. That is, a single camera view can be used to localize objects, such as particles in volume of gas. Consequently, plenoptic camera technology has recently been investigated for aerospace instrumentation including particle image velocimetry (PIV) ${ }^{8}$ and background oriented schlieren (BOS). ${ }^{9}$ Using conventional cameras in such applications, multiple cameras are used to obtain 3D information. Using plenoptic camera technology, hundreds of different 'views' are obtained by a single camera so that volumetric measurement that previously required many cameras can potentially be performed with just one (or two if highly spatially resolved measurement is required). These multiple views greatly simplify experimental setups, though the analysis of such data is computationally intensive. This paper describes the use of a plenoptic camera for optical pyrometry for the first time.

\section{Apparatus}

Figure 1 shows the schematic of the setup used for the experiment. A conventional $135 \mathrm{~mm}$ focal length lens was used to image a heated object, which was either a calibration furnace, a radiation heater or a lighted match. The lens focused light onto a microlens array which then focuses onto a CCD sensor. ${ }^{10}$ Briefly summarizing the camera specifications, the 29 MPix Bobcat 6620 Imperx camera had a 471 x 362 microlens array arranged in a hexagonal pattern. Each microlens had a focal length of 308 microns and a pitch of 77 microns. The microlens array was positioned at approximately one microlens focal length in front of the image sensor. As such, each microlens image is focused on the main lens aperture, which is effectively at infinite distance $(135+\mathrm{mm})$ compared to the focal length of the microlens. A series of three bandpass filters were placed in front of the

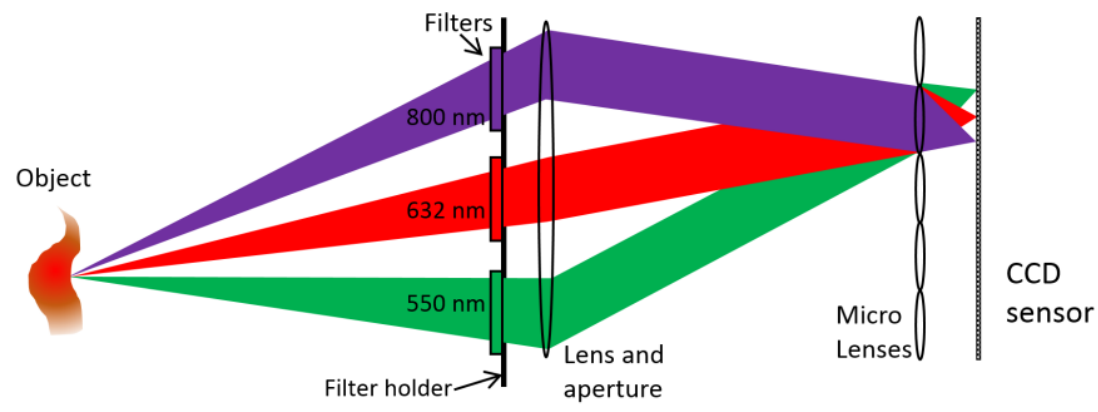

Figure 1. Detailed schematic of setup in the test section, as viewed from the top. The sample is an end-on cylinder. camera lens (see also Fig. 2).

The filters, centered at $800 \mathrm{~nm}, 632 \mathrm{~nm}$ and $550 \mathrm{~nm}$, were mounted in an opaque mount so that all the light hitting the sensor passed through one of the three filters. The three filters had $40 \mathrm{~nm}, 10 \mathrm{~nm}$ and $40 \mathrm{~nm}$ bandwidths, respectively. A neutral density (ND) 0.6 filter was placed on the $800 \mathrm{~nm}$ channel to attenuate that channel and prevent saturation of the camera, allowing longer exposure times to be acquired to obtain better balance between all three channels, thus improving signal-to-noise ratio on the red and green channels.

As shown in Fig. 1, light rays emitted from one point on the object images to different positions on the CCD camera when passing through the different colored filters. On a conventional camera, all of these light rays would be coincident on a single pixel and spectral information would be lost. But with a plenoptic camera the different colors are spatially separated on the sensor, allowing measurement of the intensity emitted by the object at different wavelengths.

The main camera lens was manually adjusted to bring the object as viewed through the lens array, into focus on the sensor. In
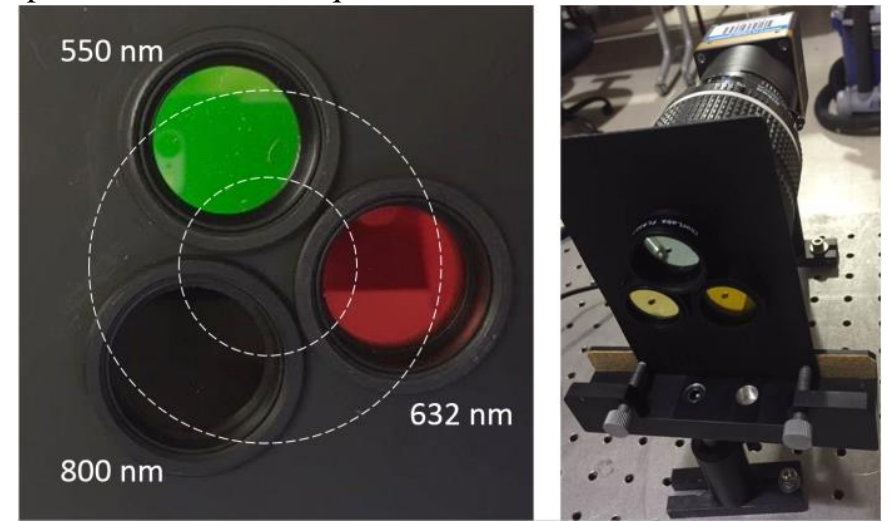

Figure 2. Image of the three bandpass filters in their mount (left) and mounted in front of the camera (right). The dashed white circles show the relative size $\sim$ of the camera lens aperture at $f / 2$ (outer circle) and f/4 (inner circle). Measurements were performed using $\mathrm{f} / 4$.

American Institute of Aeronautics and Astronautics 
principle the refocusing property ${ }^{6}$ of plenoptic cameras could be used to image objects that are not in the nominal focal plane. The system could also potentially provide range information to determine the location of the object(s) being imaged in addition to temperature. However in the current experiment this range measurement was not done. Furthermore, in the present experiment, just three filters were used, but in principle many more filters could be used to obtain more colors simultaneously. This simplicity in obtaining many colors simultaneously with a compact camera/filter arrangement is a significant advantage of the plenoptic pyrometer compared to the state of the art imaging pyrometry methods.
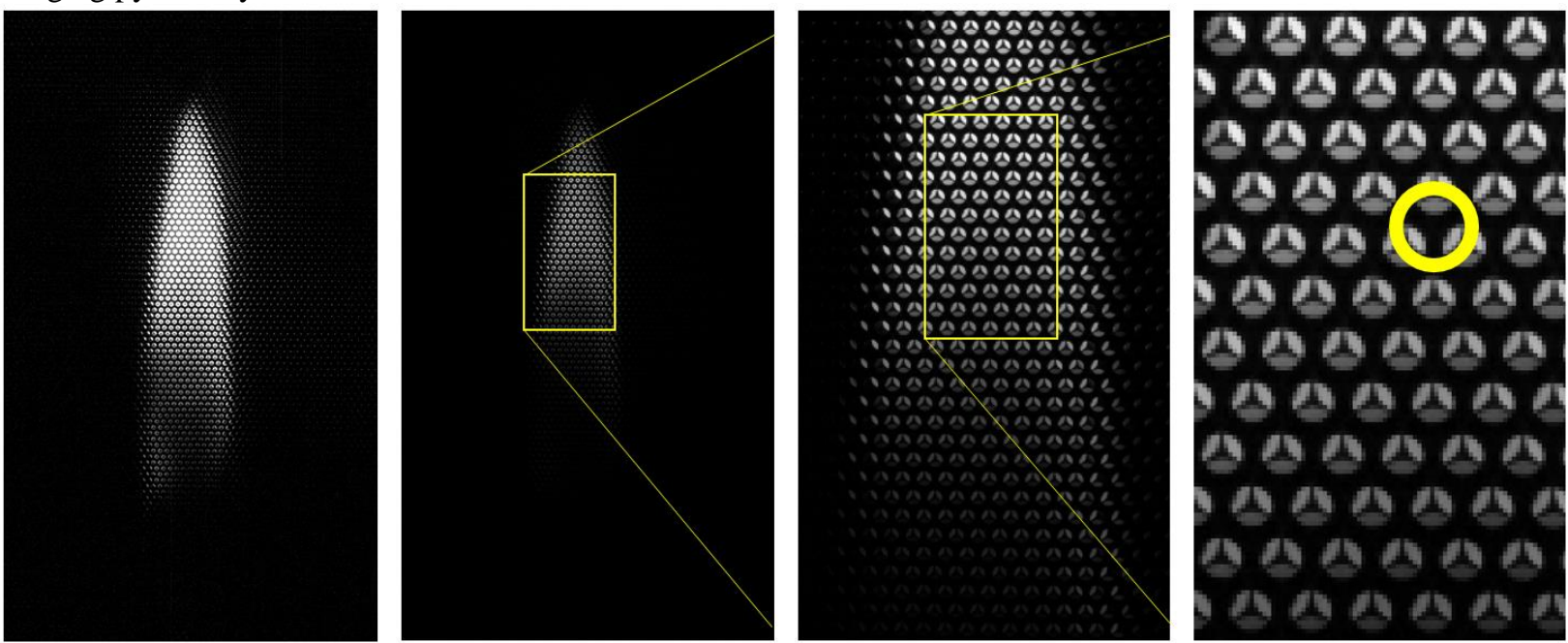

Figure 3. Plenoptic spectral images showing images obtained of a burning match.

Sample images obtained with this apparatus are shown in Fig. 3. The left panel shows the emission from a burning match imaged through the instrument with the filters in place. The intensity has been enhanced in the left panel image to show the shape of the flame. The match head can be faintly seen towards at the bottom of this image. The second panel shows the same data scaled so that the color table is not saturated. The third panel shows a magnified view of the second panel and finally the right-most panel shows an even more magnified view. The yellow circle highlights an image formed by a single micro lens.

Figure 4 compares a highly magnified plenoptic camera image with (left) and without (right) the bandpass filters present. The filter wavelengths are indicated on the left figure. If the aperture was opened further (say from $\mathrm{f} / 4$ to $\mathrm{f} / 2$ ) then the circular microlens images would overlap, rendering the images uninterpretable. This prevented detection of light through the full diameter of the bandpass filters shown
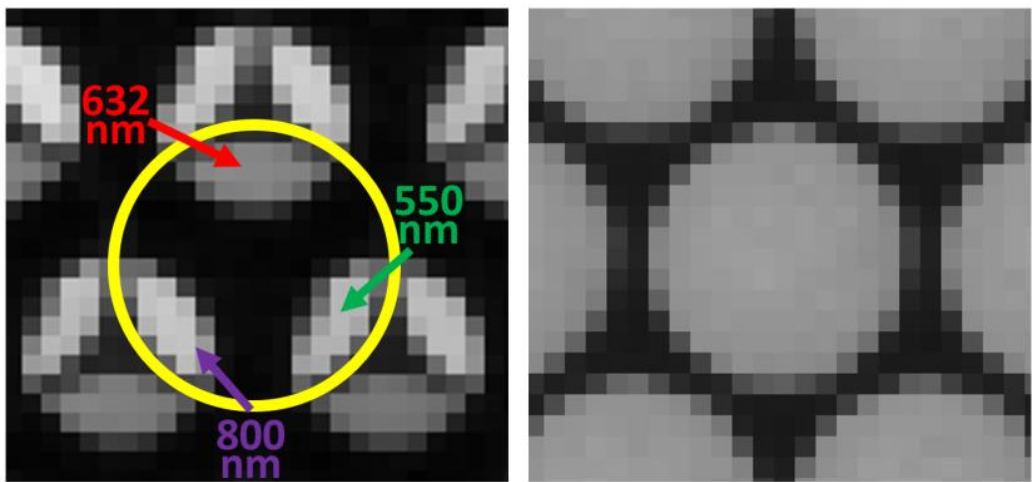

Figure 4. Comparison of images with (left) and without (right) spectral filters. in Fig. 2.

The linearity of the camera's response was measured by observing a constant intensity source and varying the duration of the camera exposure. The response was found to be nonlinear. Such nonlinearity can occur because of camera gain and lookup table settings according to the manufacturer. ${ }^{11}$ Figure 5[a] shows that it appears to be roughly logarithmic (or sqrt(x) type behavior, reminiscent of a gamma correction used in image processing), with a response that rolls off as the exposure time (corresponding to the number of incident photons in a single exposure) increases. A power law fit is shown with a dashed line, though it does not fit the data well. A $6^{\text {th }}$ order polynomial fit shown as a solid line going through the points obtains an improved fit to the measured data. In order to correct for this nonlinearity a function was needed to convert the measured counts to a corrected intensity value. Consequently, the axes on the graph were flipped and graphed on a log-log scale in Fig. $5[\mathrm{~b}]$. Again a $6^{\text {th }}$ order polynomial was fit to the data, producing an acceptable fit. The resulting polynomial shown in the figure was later 
used to convert measured counts to corrected, linearized, counts (assuming that the incident light is linear with exposure time). Note that the detector's nonlinear response is advantageous in this experiment because it helps extend the camera's dynamic range compared to a detector with a linear response.

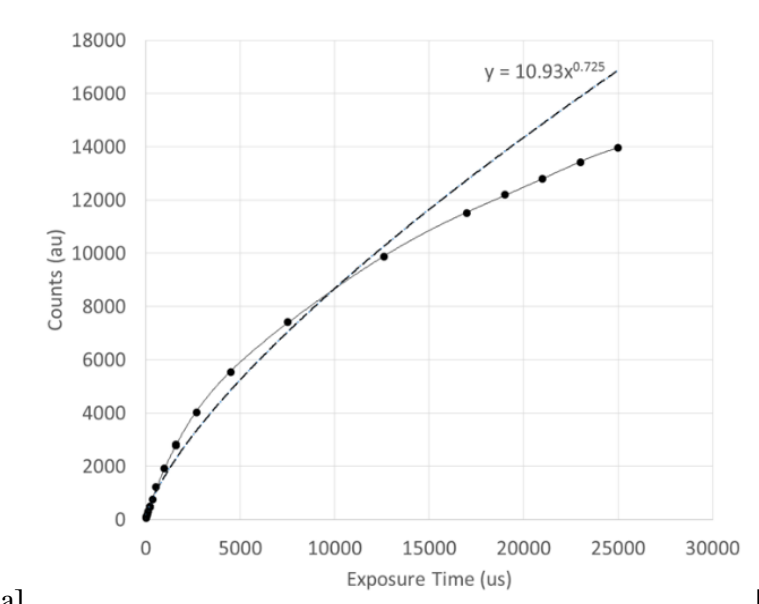

[a]

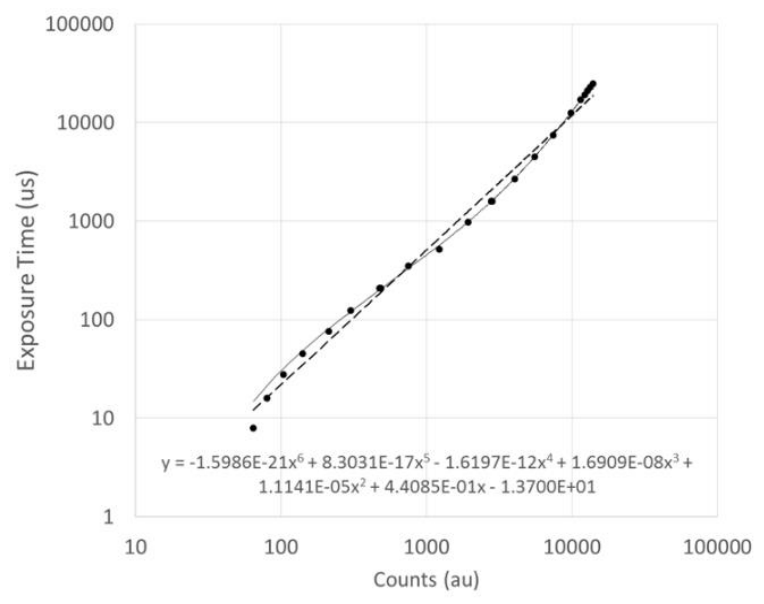

[b]

Figure 5. Camera linearity test results showing camera counts versus exposure time (left) and fit to the same data with axes reversed. Note linear scale on [a] and log-log scale on [b].

\section{Calibration and Verification}

A Mikron M360 furnace was used as a variabletemperature blackbody light source for calibration. The design of the light source produces an effective emissivity near unity (specified by the manufacturer to be 0.995). The furnace has a maximum operating temperature of $1100{ }^{\circ} \mathrm{C}(1373 \mathrm{~K})$ but the maximum temperature used in this experiment was $1050{ }^{\circ} \mathrm{C}$ $(1323 \mathrm{~K})$. The uniformity of the $25 \mathrm{~mm}$ diameter exit is within $0.1 \%$ of the control setting according to the manufacturer. Its accuracy is $\pm 1{ }^{\circ} \mathrm{C}$, and its calibration was current.

Measurement in the furnace were obtained for every $50 \mathrm{~K}$ from $973 \mathrm{~K}$ to $1323 \mathrm{~K}$. Different exposure times were used at each temperature to utilize the full dynamic range of the camera. Exposure times varied from $1.5 \mathrm{msec}$ to $0.4 \mathrm{sec}$, depending on the temperature. The mean signal intensity on each of the three color channels was corrected for exposure time and camera nonlinearity resulting in Fig. 6. Note that the $800 \mathrm{~nm}$ channel

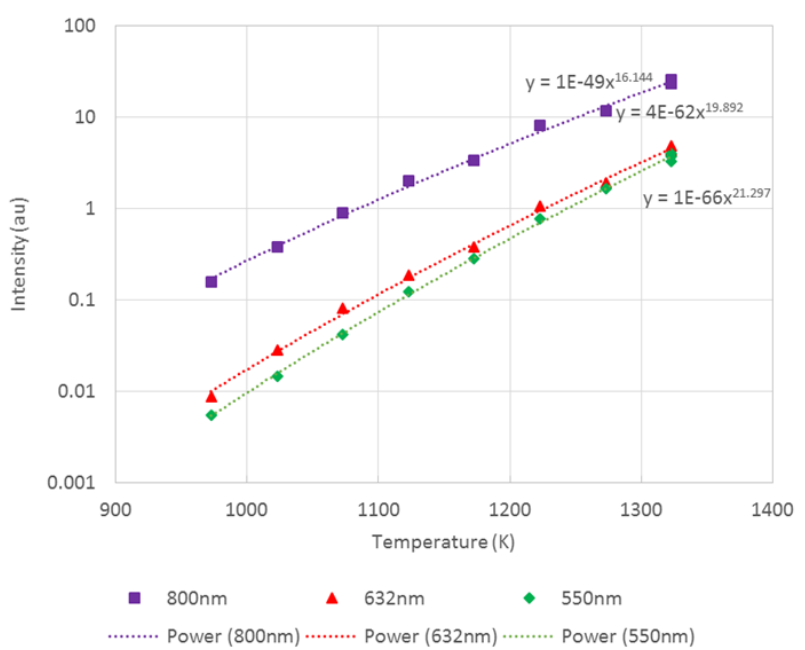

Figure 6. Intensity measured on each of the spectral channels as a function of temperature, fitted with power law equations.

has significantly higher signal compared to the others despite having an additional ND filter in series. The $632 \mathrm{~nm}$ intensity is not as high as might be expected in comparison with the other two because it has a 4-times narrower spectral bandpass width compared to the others.

It is remarkable that the power law fits show temperature exponents ranging from 16 to 21 , which is much larger than the $\mathrm{T}^{4}$ dependence predicted by Planck's law. Of course, Planck's law includes the radiation integrated over all wavelengths. The present measurement only detects specific wavelengths. So these curves include not only the overall $\mathrm{T}^{4}$ dependence but also the fact that the entire spectrum is shifting to the blue as the temperature increases.

In the subsequent analysis only the data obtained with the $800 \mathrm{~nm}$ and $550 \mathrm{~nm}$ filters were utilized because this pair of wavelengths (being widely separated in wavelength) is the most temperature-sensitive of those tested. Emission collected at these two colors is shown in comparison with the predicted emission at these wavelengths using Planck's law in Fig. 7. While a single scaling constant has been freely adjusted for best fit, the curvature of the solid line is determined a priori using Planck's law evaluated at the wavelength and temperatures shown. The 
agreement for the $800 \mathrm{~nm}$ radiation is good over the full range of temperatures. For the green channel, the agreement at high temperatures is good. At lower temperatures, ambient light appears to be interfering with the 550 $\mathrm{nm}$ channel. Consequently, temperature measurements below about $1100 \mathrm{~K}$ obtained with this system are expected to show a systematic error.

Ratios of the data points in Fig. 7 were obtained and graphed versus temperature in Fig. 8 with temperature on the vertical axis. Thus, a power law fit could be used to develop a simple relationship between measured signal intensity ratios and temperature. The resulting fit, shown as a dashed line, passes closely through the data. However, the data obtained in subsequent experiments was expected to exceed the temperature range of the calibration so the validity of an extrapolation was investigated. For comparison, Planck's law, valid over a wide temperature range, was used to compute the ratio of emission at the two colors as a function of temperature (by taking the ratio of the smooth curves in Fig. 7). This curve does not pass as well through the data, perhaps because of the room light interference at low temperatures. However both curves extrapolate similarly to higher temperatures indicating that either curve would provide similar results at higher temperatures.

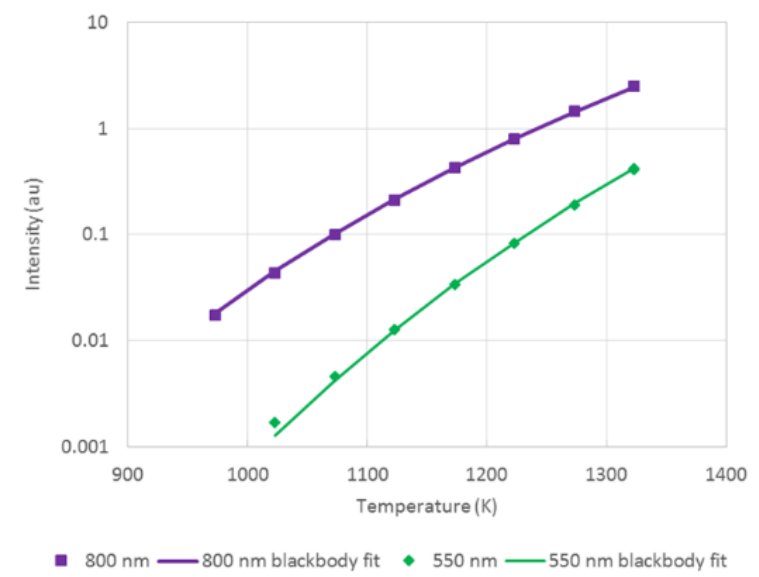

Figure 7. Comparison of blackbody theory to measurements obtained in the furnace. An overall scaling factor has been applied to the fits to calibrate the relative efficiency of the two filters.

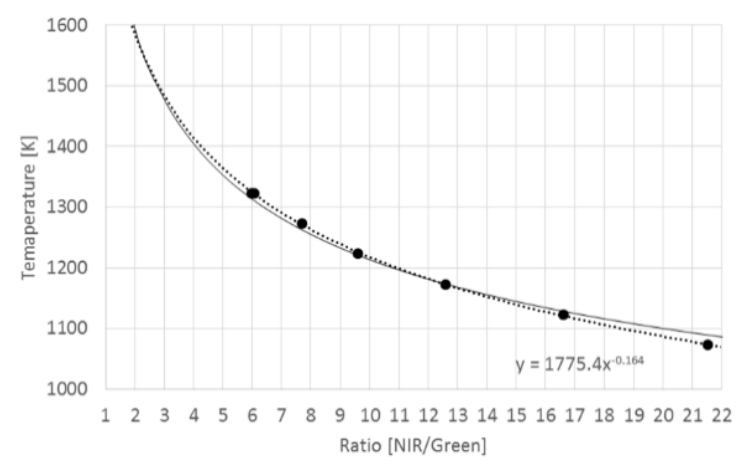

Figure 8. Relationship between the measured calibration furnace temperature and the ratio of the NIR $(800 \mathrm{~nm})$ and the green $(550 \mathrm{~nm})$ channels of the instrument. The dashed line is a best fit while the solid line uses Planck's radiation law.

After establishing a method and equations for determining temperatures from the plenoptic spectral images, the original calibration data was re-fit to determine two-dimensional temperature distributions, allowing determination of the measurement accuracy (compared to the known temperature) and precision (1- $\sigma$ variation of the temperature within the image of the uniform-temperature calibration source). Based on this secondary analysis, the system is expected to reliably measure temperatures above $1100 \mathrm{~K}$ with both an accuracy and precision of $10 \mathrm{~K}$, neglecting potentially significant errors associate with emissivity, which can vary with wavelength and temperature.

\section{Processing of Plenoptic Images}

The above analyses were obtained on raw plenoptic images like those shown in Fig. 3 after being corrected for nonlinearity and background subtraction. The imaging results shown below were obtained after post-processing the images with Auburn University's LFIT software, ${ }^{12}$ which is written in Matlab ${ }^{\mathrm{TM}}$. Only the microlens calibration and perspective view features of the software were used. A single pixel in each of the three regions shown inside the yellow circle in Fig. 4 were chosen for analysis. Unfortunately, since these regions were near the edge of the lens array image, some artifacts resulting from image reconstruction were observed in the processed images. Since these artifacts were very repeatable (they are a function of the camera and microlens setup) they could be normalized out by looking at a uniform light source (in this case, the calibration furnace). This normalization was only performed once and was applied to all the rest of the images obtained in the experiment. The uniform light source had a circular aperture of $25.4 \mathrm{~mm}$ diameter, so the correction could only be applied to data falling within this aperture.

After the images at specific wavelengths were obtained, they were further processed by the ImageJ software in a custom-written program. ${ }^{13}$ This program performed a nonlinearity correction and background subtraction and then 
the artifact correction normalization was applied. Finally, ratios of the $800 \mathrm{~nm}$ and $550 \mathrm{~nm}$ images were taken and the temperature was determined from the blackbody theory curve drawn in Fig. 8.

\section{Sample Application}

The plenoptic imaging pyrometer has been used to study an unknown temperature object as proof of concept. We obtained images of a ceramic radiation heater during steady operation and then suddenly switched off the heater. The camera acquired data at about $3 \mathrm{~Hz}$, so the cooldown of the heater could be monitored. The radiation heater is shown to operate at a steady temperature for the first 15 frames (about 5 seconds) followed by a cooldown period (Figs. 9 and 10). The signal-to-noise ratio in the temperature measurements is high when the heater is operating, but once the temperature drops below about $1100 \mathrm{~K}$ (frame 35) the signal-to-noise ratio deteriorates, mainly because of low signal in the 550-nm channel. It is evident that the dynamic range of the measurement could be improved by either using additional colored filters or by using a detector with a larger dynamic range.

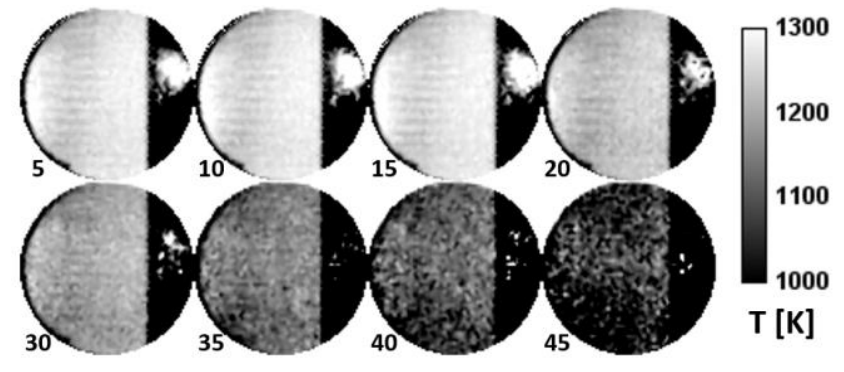

Figure 9. Temperature image of ceramic radiant heating element during steady operation (first three images in the top row) followed by cooldown. Numbers indicate frame numbers.

To demonstrate the sensitively of the instrument, Fig. $11[\mathrm{~b}]$ shows a line plot of the region highlighted in Fig. 11[a]. Spatial variations of 20 degrees are clearly observed. These features are likely related to the coiled conductor located inside the heating element. Note that no emissivity correction was made for this data set, so if the emissivity is a strong function of wavelength for this heater material then there could be an error in the temperature measurement.

[a]
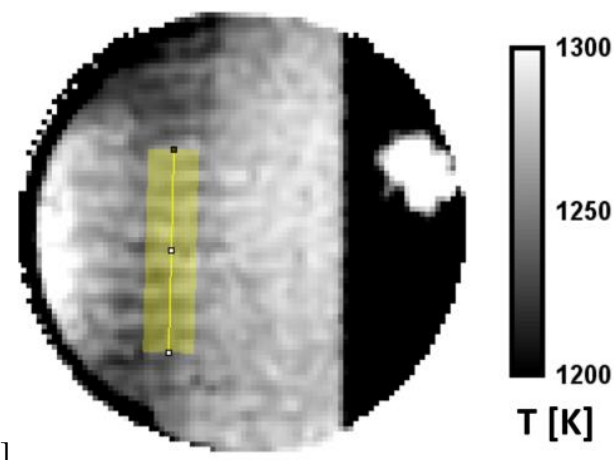

[b]
Figure 10. Average temperature measured over a uniform region in the middle of the heating element in Fig. 9 and graphed versus frame number (acquired at 3 frames per second).

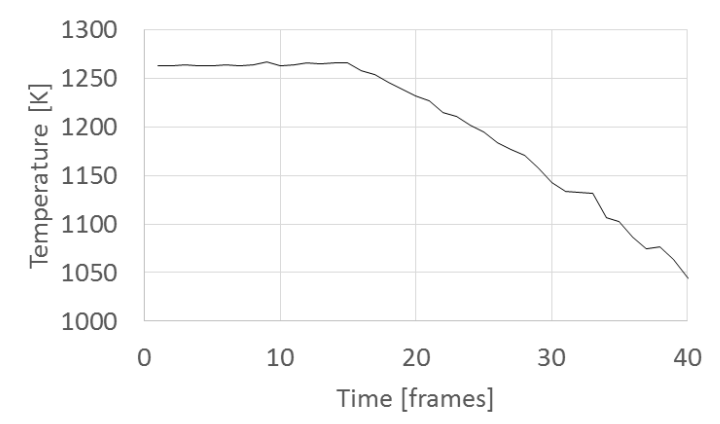

Figure 11. Detailed temperature image [a] showing the location and width of the line profile displayed in [b]. The horizontal axis of the plot is about $20 \mathrm{~mm}$ long. This data is from frame number 5. Note expanded temperature scale compared to Fig. 9.

\section{Conclusions}

A spectrally-resolving plenoptic imaging apparatus has been calibrated and demonstrated for optical pyrometry for the first time. Color bandpass filters were adapted to a plenoptic camera so that spectral information could be coded spatially on the sensor. The ratio of the intensities from the different colors was used to measure the temperature of hot objects. The sensor was first calibrated with a known-temperature light source and then was used to measure the temperature of a ceramic heater during cooldown. A limitation of the technique is that the dynamic range in temperature measurement is only a few hundred degrees, at least for one camera exposure setting. The 
dynamic range can be extended by varying the camera exposure with the current apparatus. However, in future measurements, the dynamic range could be increased by using more filters at different colors, using color filters in conjunction with ND filters, or by using a sensor with a larger dynamic range. The instrument can be upgraded to measure many different colors simultaneously by placing additional filters in the aperture plane, leading to more robust measurements with a broader dynamic range.

\section{Acknowledgements}

This work was sponsored by the NASA Langley Research Center IRAD Program through a Tier 1 effort. The authors wish to thank Ross Burns of the National Institute of Aerospace (NIA) in Hampton Virginia, Jennifer Inman of NASA Langley Research Center and Jenna Klemkowsky from Auburn University for their assistance with this work.

\section{References}

${ }^{1}$ Transactions in measurement and control, volume 1: Non-Contact Temperature Measurement, Omega Press, 1997.

${ }^{2}$ Hagen, N. and Kudenov, M.W., 2013. Review of snapshot spectral imaging technologies. Optical Engineering, 52(9), pp.090901-090901.

${ }^{3} \mathrm{Lu}$, G. and Yan, Y., 2006. Temperature profiling of pulverized coal flames using multicolor pyrometric and digital imaging techniques. IEEE transactions on instrumentation and measurement, 55(4), pp.1303-1308.

${ }^{4}$ Cavanaugh, D.B., Dombrowski, M. and Catanzaro, B., Surface Optics Corporation, 2008. Spatially corrected full-cubed hyperspectral imager. U.S. Patent 7,433,042.

${ }^{5}$ Horstmeyer, R., R. Athale and G. Euliss (2009). Modified light field architecture for reconfigurable multimode imaging, San Diego, CA, USA, SPIE.

${ }^{6} \mathrm{Ng}$, R., M. Levoy, M. Brédif, G. Duval, M. Horowitz and P. Hanrahan (2005). Light Field Photography with a Hand-Held Plenoptic Camera, Stanford Computer Science Technical Report CSTR 2 (11), 1-11

${ }^{7}$ Adelson, E. H. and J. Y. A. Wang (1992). "Single Lens Stereo with a Plenoptic Camera." IEEE Transactions on Pattern Analysis and Machine Intelligence 14(2): 99-106.

${ }^{8}$ Fahringer, T.W., Lynch, K.P. and Thurow, B.S., 2015. Volumetric particle image velocimetry with a single plenoptic camera. Measurement Science and Technology, 26(11), p.115201.

9 Klemkowsky, J.N., Thurow, B.S. and Mejia-Alvarez, R., 3D Visualization of Density Gradients Using a Plenoptic Camera and Background Oriented Schlieren Imaging, AIAA Paper 2016-1047 4-8 January 2016, San Diego, California, USA.

${ }^{10}$ Fahringer, T. W., K. P. Lynch and B. S. Thurow (2015). "Volumetric particle image velocimetry with a single plenoptic camera." Measurement Science and Technology 26(11): 115201.

${ }^{11}$ Personal Communication with Imperx technical support on 11/28/2016.

12 Bolan, J., Munz, E., Clifford, C. and Thurow, B., "Light Field Imaging Toolkit (LFIT): An Overview," SoftwareX, In Press, 2016.

13 Abràmoff, M.D., Magalhães, P.J. and Ram, S.J., 2004. Image processing with ImageJ. Biophotonics international, 11(7), pp.36-42. 